\title{
A Finite Element Model Based on Discontinuous Galerkin Methods on Moving Grids for Vertebrate Limb Pattern Formation
}

\author{
J. Zhu ${ }^{1}$, Y.-T. Zhang ${ }^{1}$, S. A. Newman ${ }^{2}$ and M. S. Alber ${ }^{1 *}$ \\ ${ }^{1}$ Department of Mathematics, University of Notre Dame, Notre Dame, IN 46556-4618, USA \\ ${ }^{2}$ Department of Cell Biology and Anatomy, Basic Science Building, \\ New York Medical College, Valhalla, NY 10595, USA
}

\begin{abstract}
Skeletal patterning in the vertebrate limb, i.e., the spatiotemporal regulation of cartilage differentiation (chondrogenesis) during embryogenesis and regeneration, is one of the best studied examples of a multicellular developmental process. Recently [Alber et al., The morphostatic limit for a model of skeletal pattern formation in the vertebrate limb, Bulletin of Mathematical Biology, 2008, v70, pp. 460-483], a simplified two-equation reaction-diffusion system was developed to describe the interaction of two of the key morphogens: the activator and an activator-dependent inhibitor of precartilage condensation formation. A discontinuous Galerkin (DG) finite element method was applied to solve this nonlinear system on complex domains to study the effects of domain geometry on the pattern generated [Zhu et al., Application of Discontinuous Galerkin Methods for reaction-diffusion systems in developmental biology, Journal of Scientific Computing, 2009, v40, pp. 391-418]. In this paper, we extend these previous results and develop a DG finite element model in a moving and deforming domain for skeletal pattern formation in the vertebrate limb. Simulations reflect the actual dynamics of limb development and indicate the important role played by the geometry of the undifferentiated apical zone.
\end{abstract}

Key Words: discontinuous Galerkin finite element methods, reaction-diffusion equations, operator splitting, triangular meshes, moving domain, complex geometry, limb development

AMS subject classification: 65M99

\footnotetext{
*Corresponding authors. E-mail addresses: malber@nd.edu (M. S. Alber), yzhang10@nd.edu (Y.-T. Zhang)
} 


\section{Introduction}

Skeletal patterning in the vertebrate limb, i.e., the spatiotemporal regulation of cartilage differentiation (chondrogenesis) during embryogenesis and regeneration, is one of the best studied examples of multicellular organogenesis $[22,15]$. Limb morphogenesis involves subcellular, cellular and supracellular components that interact in a reliable fashion to produce functional skeletal structures. Since many of the components and interactions are also typical of other embryonic processes, understanding this phenomenon can provide insights into a variety of morphogenetic events in early development.

The limb skeleton consists of nodules and rods of cartilage (later replaced by bone), arranged in tandem and parallel arrays $[16,17]$. It thus lends itself to being modeled by reaction-diffusion systems, which readily generate spot- and stripe-like patterns.

The most detailed model for vertebrate limb development presented thus far is that of [7], in which a system of eight PDEs was constructed largely on the basis of experimentally determined cellular-molecular interactions occurring in the avian and mouse limb bud. The full system has smooth solutions that exist globally in time [1] but is difficult to handle mathematically and computationally. The chemotaxis terms in the full system could cause instabilities in the numerical implementation. In particular, it is rather hard to characterize attracting stationary solutions. Moreover, biologically relevant simulations would involve a large number of experimentally justified parameters, which are often known only approximately. Recently in [2], by analytically implementing the assumption that cell differentiation relaxes faster than the evolution of the overall cell density, a simplified two-equation system was extracted from the eight-equation system governing the interaction of two of the key morphogens: the activator and an activator-dependent inhibitor of precartilage condensation formation. The reduced reaction-diffusion system has the form

$$
\begin{aligned}
& \frac{\partial C_{a}}{\partial t}=D_{a} \nabla^{2} C_{a}+U\left(C_{a}\right)-k_{a} C_{a} C_{i} \\
& \frac{\partial C_{i}}{\partial t}=D_{i} \nabla^{2} C_{i}+V\left(C_{a}\right)-k_{a} C_{a} C_{i}
\end{aligned}
$$

where $C_{a}$ denotes the concentration of the activator TGF- $\beta, C_{i}$ the concentration of the inhibitor, $D_{a}$ and $D_{i}$ the diffusion constants for the activator and the inhibitor respectively, $k_{a}$ the inhibitoractivator binding rate, $U$ and $V$ the production rates of $C_{a}$ and $C_{i}$, respectively. The system is subject to no-flux boundary conditions and zero initial concentrations for $C_{a}$ and $C_{i}$. The functions $U$ and $V$ are given by

$$
\begin{aligned}
U\left(C_{a}\right) & =\left[J_{a}^{1} \alpha\left(C_{a}\right)+J_{a}\left(C_{a}\right) \beta\left(C_{a}\right)\right] R_{e q} \\
V\left(C_{a}\right) & =J_{i}\left(C_{a}\right) \beta\left(C_{a}\right) R_{e q}
\end{aligned}
$$

where $J_{a}\left(C_{a}\right)=J_{\text {amax }}\left(C_{a} / s\right)^{n} /\left[1+\left(C_{a} / s\right)^{n}\right], J_{i}\left(C_{a}\right)=J_{\text {imax }}\left(C_{a} / \delta\right)^{q} /\left[1+\left(C_{a} / \delta\right)^{q}\right]$, and $\beta\left(C_{a}\right)=$ $\beta_{1} C_{a} /\left(\beta_{2}+C_{a}\right)$. Following [2], the parameter values in the system are taken as $D_{a}=1, D_{i}=$ $100.3, J_{\text {amax }}=6.0 \lambda, J_{\text {imax }}=8.0 \lambda, s=4.0, k_{a}=\lambda, J_{a}^{1} \alpha\left(C_{a}\right)=0.05 \lambda, \beta_{1}=0.693473, \beta_{2}=$ 
2.66294, $R_{e q}=2.0, n=q=2$. The values of the factors $\lambda, \delta$ can dramatically affect the pattern as shown in [29] and Section 3 of this paper.

Recently we developed an operator splitting discontinuous Galerkin (DG) finite element method to numerically solve the nonlinear system (1.1)-(1.2) on variable domains to study the effects of domain geometry on the pattern generated [29]. The method is based on a new DG method for solving time dependent PDEs with higher order spatial derivatives, developed by Cheng and Shu [3]. These investigators formulated the scheme by repeated integration by parts of the original equation and then replacing the interface values of the solution by carefully chosen numerical fluxes. In contrast to the local discontinuous Galerkin (LDG) method [27, 28, 10, 23, 24, 25, 26], this new DG method can be applied without introducing any auxiliary variables or rewriting the original equation in the form of a larger system, hence it is easier to formulate and implement, has a smaller effective stencil, and may reduce storage and computational cost

In this paper, we extend previous results and develop a moving grid DG finite element model on a moving and deforming domain for modeling skeletal pattern formation in the vertebrate limb. We note that an alternative way to solve reaction-diffusion systems on a moving domain with complicated geometry is to use continuous Galerkin (CG) finite element methods $[11,12,13]$. CG and DG methods each have their own advantages. CG methods have fewer degrees of freedom, especially for high spatial dimensional problems. DG methods can easily handle adaptivity strategies since refinement or coarsening of the grid can be achieved without taking into account the continuity restrictions typical of conforming finite element methods. Moreover, the degree of the approximating polynomial can be easily changed from one element to the other, and the use of general meshes with hanging nodes is allowed [4], for example there may be more than three neighbors for a triangular element. For a problem with strong hyperbolic property, such as on a fast moving domain or a convection dominated problem, DG methods can naturally incorporate the upwind numerical flux into the numerical scheme like that in the finite volume technique, to ensure the numerical stability of the computation [5]. Simulations by our DG methods in this paper reflect the authentic dynamics of limb development and the important role played by the geometry of the limb's undifferentiated apical zone in which local autoactivation-lateral inhibitory interactions occur ('LALI zone').

\section{A DG finite element model on moving grids}

The system (1.1)-(1.2) for limb development belongs to the class of reaction-diffusion systems of two chemical species. On a fixed domain, they can be written in the general form

$$
\frac{\partial u}{\partial t}=\mathbf{D} \nabla^{2} u+F(u)
$$

where $u \in \mathbf{R}^{2}$ represent concentrations of molecular species, $\mathbf{D} \in \mathbf{R}^{2 \times 2}$ is the diffusion constant matrix and it is diagonal, $\nabla^{2} u$ is the Laplacian associated with the diffusion of the molecules $u$, and $F(u)$ describes the biochemical reactions. 
To model vertebrate limb development, we consider system (2.1) on a moving domain. Let $\Omega(t)=\{(x(t), y(t))\}$ be an open, bounded, and time-dependent domain on which the reactiondiffusion system (2.1) is defined, where $(x(t), y(t))$ is a point in the domain. We triangulate $\Omega(t)$ by $\Omega_{h}(t)$ which consists of time-dependent non-overlapping triangles $\left\{\triangle_{m}(t)\right\}_{m=1}^{N}$. Let $h_{\text {min }}(t)=\min _{1 \leq m \leq N} \rho_{m}(t)$, where $\rho_{m}(t)$ is the diameter of the inscribed circle of the triangle $\triangle_{m}(t)$, and $\left\{\left(x_{i}(t), y_{i}(t)\right)\right\}_{i=1}^{M}$ denote the grid points of $\Omega_{h}(t)$. All spatial variables are functions of the temporal variable.

\subsection{Reaction-diffusion system on a moving domain}

On a moving domain, by the Reynolds transport theorem [9], system (2.1) can be extended to

$$
\frac{\mathcal{D} u}{\mathcal{D} t}+u \nabla \cdot \vec{a}=\mathbf{D} \nabla^{2} u+F(u)
$$

where $\frac{\mathcal{D} u}{\mathcal{D} t}$ is the material derivative of chemical species $u$, i.e.,

$$
\frac{\mathcal{D} u}{\mathcal{D} t}=\frac{\partial u}{\partial t}+\vec{a} \cdot \nabla u
$$

and

$$
\vec{a}(x(t), y(t), t)=\left(\frac{d x(t)}{d t}, \frac{d y(t)}{d t}\right)^{T}
$$

is the velocity of a spatial point $(x(t), y(t))$ in the moving domain.

\subsection{The DG spatial discretization on moving grids}

Define the time-dependent finite element space $V_{h}^{k}(t)=\left\{v:\left.v\right|_{\triangle_{m}(t)} \in P^{k}\left(\triangle_{m}(t)\right), m=\right.$ $1, \cdots, N\}$, where $P^{k}\left(\triangle_{m}(t)\right)$ denotes the set of all polynomials of degree at most $k$ on $\triangle_{m}(t)$.

As in [29], we formally apply the DG formulation [3] to discretize the reaction-diffusion equations (2.2) in the spatial dimensions, but keep the time variable continuous. The difference from [29] is that now the finite element space is time-dependent since we are solving the problem on a moving domain. We characterize the semi-discrete scheme as: find $u \in V_{h}^{k}(t)$, such that

$$
\begin{array}{r}
\int_{\triangle_{m}(t)} \frac{\mathcal{D} u}{\mathcal{D} t} v d x+\int_{\triangle_{m}(t)} u \nabla \cdot \vec{a} v d x-\mathbf{D} \int_{\triangle_{m}(t)} u \nabla^{2} v d x \\
+\mathbf{D} \int_{\partial_{\triangle_{m}(t)}} \widehat{u} \nabla v \cdot \vec{n}_{\partial \triangle_{m}(t)} d S-\mathbf{D} \int_{\triangle_{\triangle_{m}(t)}} v \widetilde{\nabla u} \cdot \vec{n}_{\partial \triangle_{m}(t)} d S \\
=\int_{\triangle_{m}(t)} F(u) v d x
\end{array}
$$

holds true for any $v \in V_{h}^{k}(t)$ and $m=1, \cdots, N$. The numerical fluxes on the element edges $\partial \triangle_{m}(t)$ are chosen as

$$
\widehat{u}=\frac{u^{i n}+u^{e x t}}{2}
$$




$$
\widetilde{\nabla u}=\frac{(\nabla u)^{i n}+(\nabla u)^{e x t}}{2}+\beta[u]
$$

where the jump term

$$
[u]=\left.\left(u^{e x t}-u^{i n}\right)\right|_{\partial \triangle_{m}(t)} \cdot \vec{n}_{\partial \triangle_{m}(t)},
$$

$u^{i n}$ and $u^{e x t}$ are the limits of $u$ at $x \in \partial \triangle_{m}(t)$ taken from the interior and the exterior of $\triangle_{m}(t)$ respectively, $\vec{n}_{\partial \triangle_{m}(t)}$ is the outward unit normal to the element $\triangle_{m}(t)$ at $x \in \partial \triangle_{m}(t)$, and $\beta$ is a positive quantity that is of the order $\mathcal{O}\left(h_{\min }^{-1}(t)\right)$. Following [3], we take $\beta=10 / h_{\min }(t)$. The choice of numerical fluxes (2.5)-(2.7) is crucial for the stability and convergence of the DG scheme (2.4). See [6,3] for further discussion of the choice of numerical fluxes.

Following [29], we use the Strang type second-order symmetrical operator splitting schemes $[19,8]$ to avoid solving the completely coupled nonlinear system from the fully implicit temporal discretization and overcome the computational challenge from the stiffness of reaction-diffusion equations (2.2) and the DG spatial discretization operator. We consider the $P^{1}$ case in this paper such that the order of accuracy in the spatial direction corresponds to the splitting error order in the temporal direction, and they are both 2 .

As a straightforward example, we describe the detailed numerical formulae for the scalar case of (2.4). The corresponding system case can be solved component by component using similar formulae. For each element $\triangle_{m}(t)$, denote its three neighboring elements by $i_{m}, j_{m}$, and $k_{m}$. To simplify notations in the following presentation, we will omit the subscript $m$ and just use $i, j, k$ to represent the neighboring cells of $\triangle_{m}(t)$. Since limb development is accompanied by moderate growth, the apical zone of the limb bud does not deform rapidly. Hence in this computational model the mesh movement is controlled such that there is no degenerate element formed during movement of the domain. Therefore the neighboring elements of each element do not merge and the indexes $i, j, k$ for neighboring elements are time-independent. The linear polynomial on $\triangle_{m}(t)$ is represented by

$$
u(x, y, t)=a_{m}(t)+b_{m}(t) \xi_{m}(x(t), y(t), t)+c_{m}(t) \eta_{m}(x(t), y(t), t),
$$

where $\xi_{m}$ and $\eta_{m}$ are time-dependent local basis functions on $\triangle_{m}(t)$

$$
\begin{aligned}
\xi_{m}(x(t), y(t), t) & =\frac{x(t)-x_{m}(t)}{h_{m}(t)}, \\
\eta_{m}(x(t), y(t), t) & =\frac{y(t)-y_{m}(t)}{h_{m}(t)},
\end{aligned}
$$

and $\left(x_{m}(t), y_{m}(t)\right)$ is the barycenter of the element $\triangle_{m}(t), h_{m}(t)=\sqrt{\left|\Delta_{m}(t)\right|}$ with $\left|\Delta_{m}(t)\right|$ denoting the area of $\triangle_{m}(t)$. The movement of $\triangle_{m}(t)$ and the whole mesh $\Omega_{h}(t)$ are pre-determined by the development of the LALI zone of limb bud.

By taking $v=1, \xi_{m}, \eta_{m}$ on $\triangle_{m}(t)$ and $v=0$ elsewhere, the DG formulation (2.4) is converted 
from the integral form to the following system, for $m=1, \cdots, N$ :

$$
\begin{aligned}
& p_{11}(t) a_{m}^{\prime}(t)+p_{12}(t) b_{m}^{\prime}(t)+p_{13}(t) c_{m}^{\prime}(t)+ \\
& q_{11}(t) a_{m}(t)+q_{12}(t) b_{m}(t)+q_{13}(t) c_{m}(t)+ \\
& k_{11}(t) a_{m}(t)+k_{12}(t) b_{m}(t)+k_{13}(t) c_{m}(t)= \\
& \mathbf{D}\left\{w_{a m 1}(t) a_{m}(t)+w_{b m 1}(t) b_{m}(t)+w_{c m 1}(t) c_{m}(t)+\right. \\
& \left.\sum_{l=i, j, k}\left[w_{a l 1}(t) a_{l}(t)+w_{b l 1}(t) b_{l}(t)+w_{c l 1}(t) c_{l}(t)\right]\right\}+ \\
& \left(p_{11}(t) / 3\right) \sum_{l=i, j, k} F\left(u\left(x_{m, l}(t), y_{m, l}(t)\right)\right), \\
& p_{21}(t) a_{m}^{\prime}(t)+p_{22}(t) b_{m}^{\prime}(t)+p_{23}(t) c_{m}^{\prime}(t)+ \\
& q_{21}(t) a_{m}(t)+q_{22}(t) b_{m}(t)+q_{23}(t) c_{m}(t)+ \\
& k_{21}(t) a_{m}(t)+k_{22}(t) b_{m}(t)+k_{23}(t) c_{m}(t)= \\
& \mathbf{D}\left\{w_{a m 2}(t) a_{m}(t)+w_{b m 2}(t) b_{m}(t)+w_{c m 2}(t) c_{m}(t)+\right. \\
& \left.\sum_{l=i, j, k}\left[w_{a l 2}(t) a_{l}(t)+w_{b l 2}(t) b_{l}(t)+w_{c l 2}(t) c_{l}(t)\right]\right\}+ \\
& \left(p_{11}(t) / 3\right) \sum_{l=i, j, k} F\left(u\left(x_{m, l}(t), y_{m, l}(t)\right)\right) \xi_{m}\left(x_{m, l}(t), y_{m, l}(t), t\right), \\
& p_{31}(t) a_{m}^{\prime}(t)+p_{32}(t) b_{m}^{\prime}(t)+p_{33}(t) c_{m}^{\prime}(t)+ \\
& q_{31}(t) a_{m}(t)+q_{32}(t) b_{m}(t)+q_{33}(t) c_{m}(t)+ \\
& k_{31}(t) a_{m}(t)+k_{32}(t) b_{m}(t)+k_{33}(t) c_{m}(t)= \\
& \mathbf{D}\left\{w_{a m 3}(t) a_{m}(t)+w_{b m 3}(t) b_{m}(t)+w_{c m 3}(t) c_{m}(t)+\right. \\
& \left.\sum_{l=i, j, k}\left[w_{a l 3}(t) a_{l}(t)+w_{b l 3}(t) b_{l}(t)+w_{c l 3}(t) c_{l}(t)\right]\right\}+ \\
& \left(p_{11}(t) / 3\right) \sum_{l=i, j, k} F\left(u\left(x_{m, l}(t), y_{m, l}(t)\right)\right) \eta_{m}\left(x_{m, l}(t), y_{m, l}(t), t\right),
\end{aligned}
$$

where the coefficients $\left\{p_{r s}\right\}_{r, s=1}^{3},\left\{q_{r s}\right\}_{r, s=1}^{3},\left\{k_{r s}\right\}_{r, s=1}^{3},\left\{\left\{w_{\text {alr }}\right\}_{r=1}^{3},\left\{w_{b l r}\right\}_{r=1}^{3},\left\{w_{c l r}\right\}_{r=1}^{3}\right\}_{l=m, i, j, k}$ depend on the local geometry of the mesh (i.e., triangle $\triangle_{m}(t)$ and its neighboring cells $i, j, k$ and $\left.\vec{n}_{\partial \triangle_{m}(t)}\right)$, the local basis functions $1,\left\{\xi_{l}(x, y, t), \eta_{l}(x, y, t)\right\}_{l=m, i, j, k}$, and $\beta .\left\{x_{m, l}(t), y_{m, l}(t)\right\}_{l=i, j, k}$ are the mid-points of the three edges $\left\{e_{l}\right\}_{l=i, j, k}$ of $\triangle_{m}(t)$ which serve as Gaussian quadrature points for the integral involving the nonlinear reaction terms in (2.4). The detailed formulae for computing these coefficients are presented in the Appendix.

We rewrite equations (2.11)-(2.13) to the matrix-vector form

$$
P_{m}(t) \vec{V}_{m}^{\prime}(t)+Q_{m}(t) \vec{V}_{m}(t)+K_{m}(t) \vec{V}_{m}(t)=\mathbf{D} \sum_{l=m, i, j, k} W_{l}(t) \vec{V}_{l}(t)+\vec{F}_{m}\left(\vec{V}_{m}(t)\right) .
$$


where

$$
\begin{aligned}
& \vec{V}_{m}(t)=\left(\begin{array}{c}
a_{m}(t) \\
b_{m}(t) \\
c_{m}(t)
\end{array}\right), \vec{V}_{l}(t)=\left(\begin{array}{c}
a_{l}(t) \\
b_{l}(t) \\
c_{l}(t)
\end{array}\right), P_{m}(t)=\left(\begin{array}{lll}
p_{11}(t) & p_{12}(t) & p_{13}(t) \\
p_{21}(t) & p_{22}(t) & p_{23}(t) \\
p_{31}(t) & p_{32}(t) & p_{33}(t)
\end{array}\right), \\
& Q_{m}(t)=\left(\begin{array}{lll}
q_{11}(t) & q_{12}(t) & q_{13}(t) \\
q_{21}(t) & q_{22}(t) & q_{23}(t) \\
q_{31}(t) & q_{32}(t) & q_{33}(t)
\end{array}\right), K_{m}(t)=\left(\begin{array}{lll}
k_{11}(t) & k_{12}(t) & k_{13}(t) \\
k_{21}(t) & k_{22}(t) & k_{23}(t) \\
k_{31}(t) & k_{32}(t) & k_{33}(t)
\end{array}\right), \\
& W_{l}(t)=\left(\begin{array}{lll}
w_{a l 1}(t) & w_{b l 1}(t) & w_{c l 3}(t) \\
w_{a l 2}(t) & w_{b l 2}(t) & w_{c l 2}(t) \\
w_{a l 3}(t) & w_{b l 3}(t) & w_{c l 3}(t)
\end{array}\right) \\
& \vec{F}_{m}\left(\vec{V}_{m}(t)\right)=p_{11}(t) / 3\left(\begin{array}{c}
\sum_{l=i, j, k} F\left(u\left(x_{m, l}(t), y_{m, l}(t)\right)\right) \\
\sum_{l=i, j, k} F\left(u\left(x_{m, l}(t), y_{m, l}(t)\right)\right) \xi_{m}\left(x_{m, l}(t), y_{m, l}(t), t\right) \\
\sum_{l=i, j, k} F\left(u\left(x_{m, l}(t), y_{m, l}(t)\right)\right) \eta_{m}\left(x_{m, l}(t), y_{m, l}(t), t\right)
\end{array}\right) .
\end{aligned}
$$

Finally we have the ODE system resulting from the DG spatial discretization:

$$
\begin{aligned}
\vec{V}_{m}^{\prime}(t) & =P_{m}(t)^{-1}\left[\left(\mathbf{D} W_{m}(t)-Q_{m}(t)-K_{m}(t)\right) \vec{V}_{m}(t)+\mathbf{D} \sum_{l=i, j, k} W_{l}(t) \vec{V}_{l}(t)\right] \\
& +P_{m}(t)^{-1} \vec{F}_{m}\left(\vec{V}_{m}(t)\right) .
\end{aligned}
$$

\subsection{Temporal discretization}

The ODE (2.15) has a linear term resulting from the diffusion and domain movement and a nonlinear term coming from the reaction expression in (2.2). Both of these terms can cause stiffness in the reaction-diffusion system and present challenges for temporal discretization schemes. Hence we need to use fully implicit schemes to solve (2.15). In order to avoid solving a large coupled nonlinear system of equations at every time step, we adopted the trapezoidal operator splitting (OS) scheme [8], which belongs to the class of Strang type second-order symmetrical operator splitting schemes [19], to split the linear terms from the nonlinear terms of (2.15). The large nonlinear problem is decoupled, and hence we can solve the linear part and the nonlinear part individually by implicit temporal schemes. The resulting nonlinear problems are local for each element and they can be solved efficiently by an iterative scheme such as Newton's method.

We denote the numerical solution of the ODE system (2.15) at $t=t^{n}$ by $\vec{V}_{m}^{n}$. To evolve the system from step $t^{n}$ to $t^{n+1}$, we apply the trapezoidal OS scheme for (2.15):

Step 1 - apply forward Euler method for the linear term at $\left[t^{n}, t^{n+\frac{1}{2}}\right]$,

$$
\begin{aligned}
& \vec{v}_{0, m}=\vec{V}_{m}^{n} \\
& \vec{v}_{1, m}=\vec{v}_{0, m}+\frac{1}{2} \Delta t P_{m}\left(t^{n}\right)^{-1}\left[\left[\mathbf{D} W_{m}\left(t^{n}\right)-Q_{m}\left(t^{n}\right)-K_{m}\left(t^{n}\right)\right] \vec{v}_{0, m}+\mathbf{D} \sum_{l=i, j, k} W_{l}\left(t^{n}\right) \vec{v}_{0, l}\right] \\
& m=1, \cdots, N
\end{aligned}
$$


Step 2 - apply Crank-Nicholson method for the nonlinear term at $\left[t^{n}, t^{n+1}\right]$, with $\vec{v}_{1, m}$ as input data:

$$
\vec{v}_{2, m}=\vec{v}_{1, m}+\frac{1}{2} \triangle t\left[P_{m}\left(t^{n}\right)^{-1} \overrightarrow{\widetilde{F}}_{m}\left(\vec{v}_{1, m}\right)+P_{m}\left(t^{n+1}\right)^{-1} \overrightarrow{\widetilde{F}}_{m}\left(\vec{v}_{2, m}\right)\right] .
$$

The local nonlinear system (2.17) on the element $m$ is solved by Newton iterations, with the initial guess $\vec{v}_{1, m}$, for $m=1, \cdots, N$.

Step 3 - apply backward Euler method for the linear term at $\left[t^{n+\frac{1}{2}}, t^{n+1}\right]$, with $\vec{v}_{2, m}$ as input data,

$$
\begin{aligned}
\vec{v}_{3, m}= & \vec{v}_{2, m}+\frac{1}{2} \Delta t P_{m}\left(t^{n+1}\right)^{-1} \\
& {\left[\left[\mathbf{D} W_{m}\left(t^{n+1}\right)-Q_{m}\left(t^{n+1}\right)-K_{m}\left(t^{n+1}\right)\right] \vec{v}_{3, m}+\mathbf{D} \sum_{l=i, j, k} W_{l}\left(t^{n+1}\right) \vec{v}_{3, l}\right] } \\
\vec{V}_{m}^{n+1}= & \vec{v}_{3, m}, \quad m=1, \cdots, N .
\end{aligned}
$$

The sparse linear system (2.18) is solved by the sparse linear solver "lin_sol_gen_coordinate"of the IMSL package.

\subsection{Algorithm convergence analysis for a system with the exact solution}

In this Section, we perform a numerical convergence analysis of the scheme for solving a parabolic PDE on a moving and growing domain with an exact solution.

Example. Consider the two-dimensional nonlinear problem

$$
\left\{\begin{array}{l}
\frac{D u}{D t}+u \nabla \cdot \vec{a}=\nabla^{2} u-u^{2}+e^{-2} \cos (\pi x)^{2} \cos (\pi y)^{2}+\left(2 \pi^{2}-1\right) e^{-t} \cos (\pi x) \cos (\pi y) \\
-e^{-t} \pi \sin (\pi x) \cos (\pi y) x^{\prime}-e^{-t} \pi \cos (\pi x) \sin (\pi y) y^{\prime}+\left(\sigma_{x}+\sigma_{y}\right) e^{-t} \cos (\pi x) \cos (\pi y) \\
\quad \quad(x, y) \in(0,1) \times(0,1) \\
\vec{a}=\left(x^{\prime}, y^{\prime}\right)^{T}=\left(\sigma_{x} x, \sigma_{y} y\right)^{T}, \\
u(x, y, 0)=\cos (\pi x) \cos (\pi y)
\end{array}\right.
$$

with no flux boundary conditions. The initial domain is $(0,1) \times(0,1)$. For any $(x, y), x^{\prime}=\sigma_{x} x$ and $y^{\prime}=\sigma_{y} y$. Thus, at $T=1$, the domain changes to $\left(0, e^{\sigma_{x}}\right) \times\left(0, e^{\sigma_{y}}\right)$. In this problem, we take $\sigma_{x}=\sigma_{y}=0.5$. The exact solution is $u(x, y, t)=e^{-t} \cos (\pi x) \cos (\pi y)$ where $x$ and $y$ are both functions of the time variable. The simulation is carried up to $T=1.0$. We perform the numerical convergence analysis on successively refined meshes. The coarsest mesh is shown in Figure 1(a). The refinement of the meshes is done in a uniform way, namely by cutting each triangle into four smaller similar ones. The $L^{1}, L^{2}$ and $L^{\infty}$ errors, order of accuracy and CPU times are measured and listed in Table 1. The time step size is taken to be $\Delta t=0.1 h_{\min }(0)$. The second order accuracy in Table 1 has the expected values. 
Table 1: CPU time, error, and order of accuracy of the DG-trapezoidal OS scheme for the example in Section 2.4. Final time $T=1.0$.

\begin{tabular}{|c|c|cc|cc|cc|}
\hline \# of Cells & CPU(s) & $L^{1}$ error & order & $L^{2}$ error & order & $L^{\infty}$ error & order \\
\hline 44 & 0.43 & $5.13 \mathrm{E}-02$ & - & $4.15 \mathrm{E}-02$ & - & $7.99 \mathrm{E}-02$ & - \\
\hline 176 & 4.32 & $1.39 \mathrm{E}-02$ & 1.88 & $1.13 \mathrm{E}-02$ & 1.87 & $2.26 \mathrm{E}-02$ & 1.82 \\
\hline 704 & 95.54 & $3.57 \mathrm{E}-03$ & 1.96 & $2.91 \mathrm{E}-03$ & 1.96 & $6.02 \mathrm{E}-03$ & 1.90 \\
\hline 2816 & 3975 & $9.04 \mathrm{E}-04$ & 1.98 & $7.36 \mathrm{E}-04$ & 1.98 & $1.55 \mathrm{E}-03$ & 1.96 \\
\hline 11264 & 258405 & $2.28 \mathrm{E}-04$ & 1.99 & $1.85 \mathrm{E}-04$ & 1.99 & $3.92 \mathrm{E}-04$ & 1.99 \\
\hline
\end{tabular}

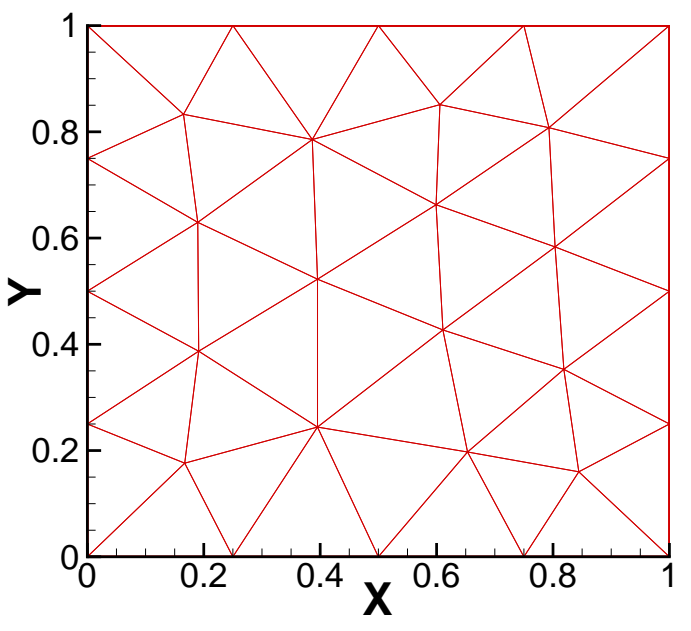

(a)

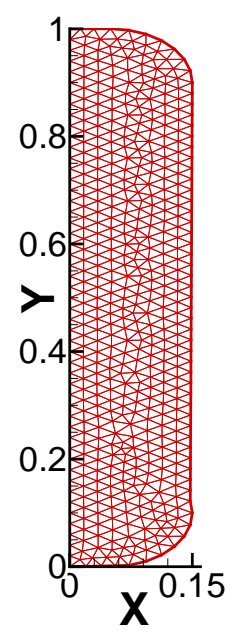

(b)

Figure 1: Meshes in the numerical simulations of Sections 2 and 3. (see text) 


\section{Reaction-diffusion mechanism for limb skeletal pattern for- mation}

The computational mesh at $t=0$ is shown in Figure 1(b). There are two color scales in the simulation pictures (Fig. 2 (a)-(f)), blue-green-red scale, representing the activator morphogen concentration in the LALI zone in which we solve the reaction-diffusion system (1.1) and (1.2), and grayscale, representing formed skeletal elements of the "frozen zone") (see [7] and [16]). At the very beginning there is only a LALI zone. The LALI zone grows at a constant rate, but due to the decline in potency of the apical ectodermal ridge (reviewed in [16]), it also shinks in the simulation in the $x$ direction. The overall result is a time-dependent reduction in the width of the LALI zone, as seen in the developing limb [20]. The moving velocity of the LALI zone is determined by the ratio of the sizes of LALI zone at different stages, and it is approximated by $x^{\prime}(t)=\sigma_{x}(x-t)+1$ with $\sigma_{x}=-0.2896$. The size of the LALI zone in the $y$ direction stays the same in the simulation, i.e., $y^{\prime}(t)=0$. The frozen zone does not move but its size grows at the speed of $v=1$ due to the cell condensation of the LALI zone. At every 0.05 unit of time, we copy the concentration values on the computation grids of the left boundary of the LALI zone to the new grid points in the frozen zone. From $t=0$ to $1.4, \lambda=1500$ and $\delta=4.7$. From $t=1.4$ to $2.4, \lambda=5000$ and $\delta=4.9$. From $t=2.4$ to $3.0, \lambda=16500$ and $\delta=4.9$. The final time is 3.0 and the time step size $\Delta t$ is $2 \times 10^{-5}$ in the simulations. The pattern arises in a proximodistal fashion as seen in amniote (lizard, bird and mammal) limbs, and the final form is similar to the 3-digit chicken wing.

We justify the change of parameters at the different phases of the simulation on the basis of the key role played by the dramatic changes in the distributions of Hox gene products in the apical zone at the different phases of limb development [14]. The Hox proteins are transcription factors that regulate the levels of developmentally important signals such as the activating and inhibitory morphogens of our model [21]. Although "recombinant" limb buds with disrupted Hox protein gradients can form limb skeletons with discrete jointed elements, the skeletons are grossly abnormal $[30,18]$. In the simulations shown in Fig. 3, the parameter value of $\lambda$ is changed to $\lambda=17000$ after time $T=2.40$ when the two-digit has been formed. The final pattern under these conditions has 4 digits, like the chicken leg.

\section{Conclusion}

The computational strategy based on a discontinuous Galerkin finite element method and employed in this paper for simulating a reaction-diffusion system in a moving and deforming domain of nonsymmetrical shape, is both novel and numerically advanced. In biological terms, however, it has enabled the confirmation, in principle of a simple, experimentally based mechanism for the proximodistal increase in the number of skeletal elements during vertebrate limb development. This basic framework should be extendable to 3 dimensions and imposition of nonuniform response functions across the various limb axes will permit the formulation of hypotheses for the individuation of skeletal elements and associated pattern asymmetries. 


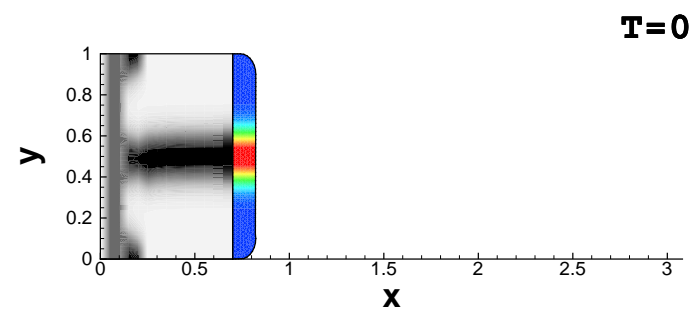

(a)

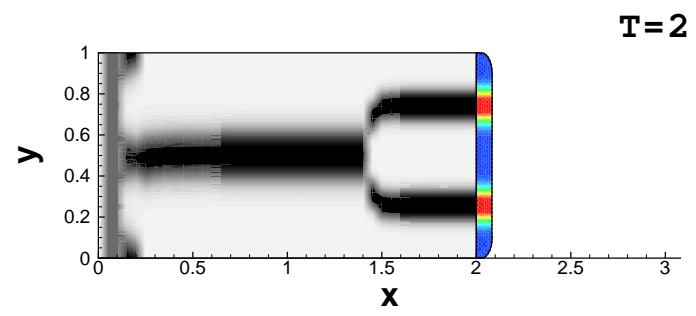

(c)

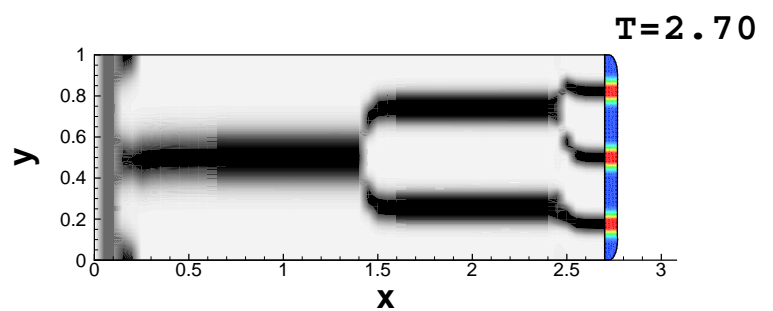

(e)

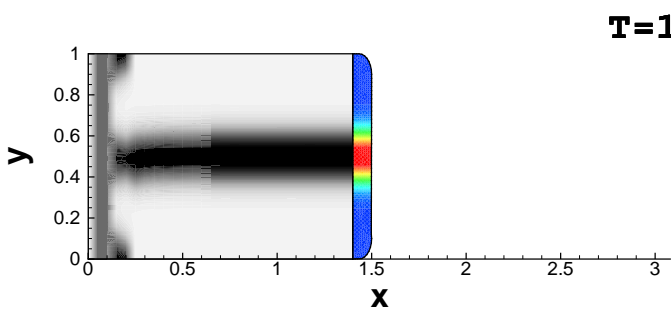

(b)

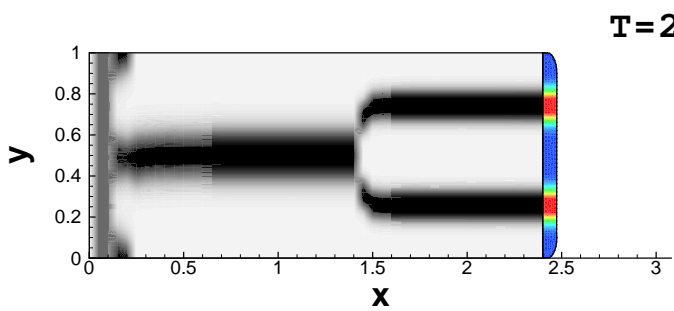

(d)

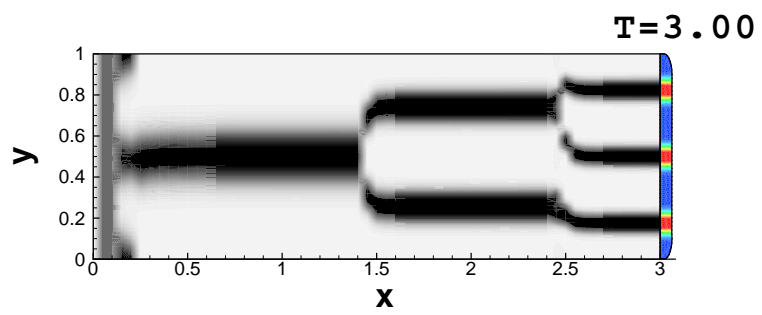

(f)

Figure 2: Patterns of activator morphogen (color scale) and formed skeletal elements (gray scale) at various times of development in the model limb, using standard parameter values. Color legend: red corresponds to 5.0, green to 2.5, blue to 0.0. (a) at time $T=0.70$; (b) at time $T=1.40$; (c) at time $T=2.00$; (d) at time $T=2.40$; (e) at time $T=2.70$; (f) at time $T=3.00$. Stages shown correspond to chicken forelimb development between 3.5 and 7 days of incubation. 


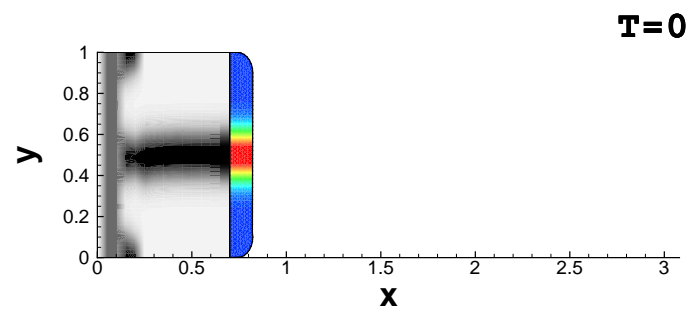

(a)

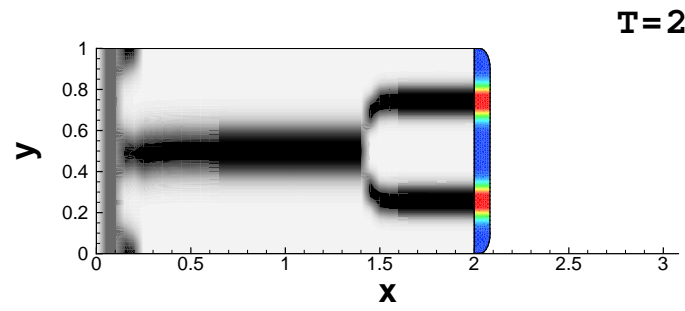

(c)

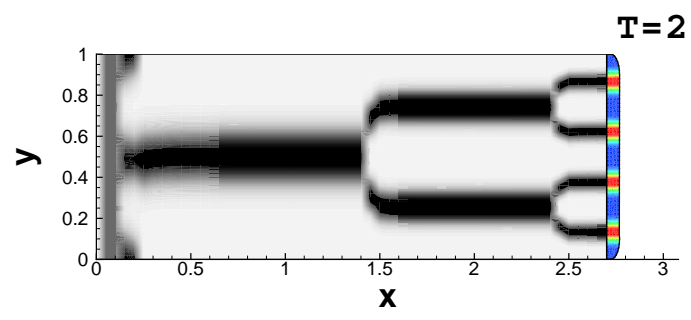

(e)

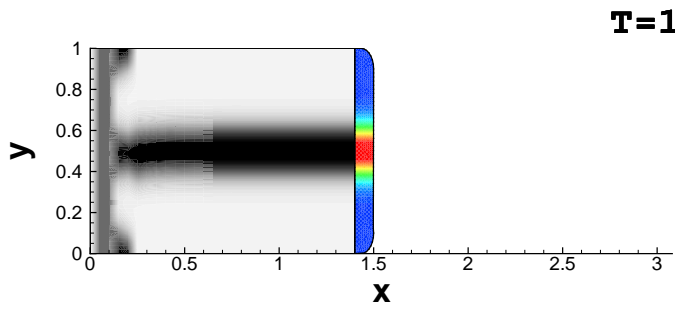

(b)

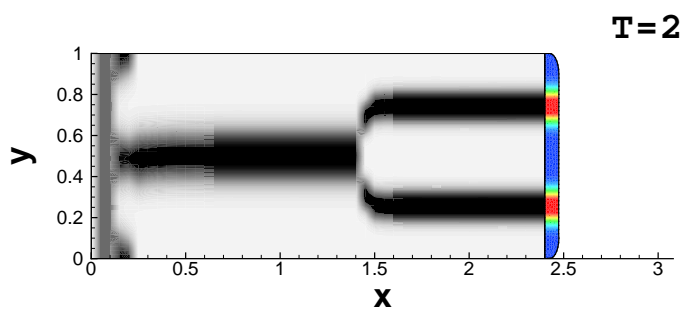

(d)

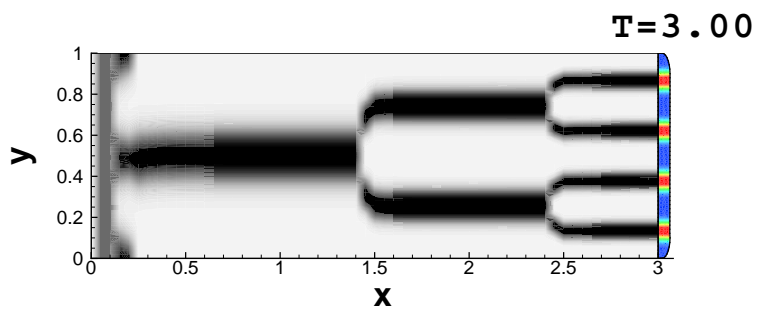

(f)

Figure 3: Patterns of activator morphogen (color scale) and formed skeletal elements (gray scale) at various times of development in the model limb, using standard parameter values except that $\lambda=17000$ after time $T=2.40$. Color legend: red corresponds to 5.0, green to 2.5, blue to 0.0. (a) at time $T=0.70$; (b) at time $T=1.40$; (c) at time $T=2.00$; (d) at time $T=2.40$; (e) at time $T=2.70$; (f) at time $T=3.00$. Stages shown correspond to chicken hindlimb development between 3.5 and 7 days of incubation. 


\section{Acknowledgements}

The research of Y.-T. Zhang is partially supported by NSF grant DMS-0810413 and Oak Ridge Associated Universities (ORAU) Ralph E. Powe Junior Faculty Enhancement Award. S.A. Newman acknowledges support from NSF grant FIBR-0526854. M. Alber was partially supported by the NSF grant DMS-0719895.

\section{Appendix: Formulae for Mesh-Dependent Constants in Equa- tion $(2.15)$}

Denote $\frac{d x}{d t}$ as $x^{\prime}$. Then

$$
\begin{aligned}
x_{m}(t) & =\frac{x_{i}(t)+x_{j}(t)+x_{k}(t)}{3}, \\
y_{m}(t) & =\frac{y_{i}(t)+y_{j}(t)+y_{k}(t)}{3} . \\
x_{m}^{\prime} & =\frac{x_{i}^{\prime}+x_{j}^{\prime}+x_{k}^{\prime}}{3}, \\
y_{m}^{\prime} & =\frac{y_{i}^{\prime}+y_{j}^{\prime}+y_{k}^{\prime}}{3} .
\end{aligned}
$$

Let

$$
s s=\operatorname{det}\left(\begin{array}{ccc}
x_{i} & x_{j} & x_{k} \\
y_{i} & y_{j} & y_{k} \\
1 & 1 & 1
\end{array}\right)
$$

We have

$$
\begin{aligned}
\left|\Delta_{m}(t)\right| & =|s s| / 2, \\
h_{m} & =\sqrt{\frac{1}{2}|s s|} \\
h_{m}^{\prime} & =\frac{1}{2 \sqrt{2}} \frac{(|s s|)^{\prime}}{\sqrt{|s s|}}= \begin{cases}\frac{s s^{\prime}}{4 h_{m}} & \text { if } s s>=0 \\
\frac{-s s^{\prime}}{4 h_{m}} & \text { if } s s<0\end{cases}
\end{aligned}
$$

and

$$
\begin{gathered}
\beta=10 \cdot \min _{m=1, \cdots, N} \frac{2|s s|}{\left|e_{i}\right|+\left|e_{j}\right|+\left|e_{k}\right|} . \\
\xi_{m}^{\prime}=\frac{\left(x^{\prime}-x_{m}^{\prime}\right) h_{m}-\left(x-x_{m}\right) h_{m}^{\prime}}{h_{m}^{2}}, \\
\eta_{m}^{\prime}=\frac{\left(y^{\prime}-y_{m}^{\prime}\right) h_{m}-\left(y-y_{m}\right) h_{m}^{\prime}}{h_{m}^{2}} \\
u_{m}^{\prime}=a_{m}^{\prime}+b_{m}^{\prime} \xi_{m}+c_{m}^{\prime} \eta_{m}+b_{m} \xi_{m}^{\prime}+c_{m} \eta_{m}^{\prime} .
\end{gathered}
$$


The matrix $P_{m}(t)=\left(\begin{array}{lll}p_{11}(t) & p_{12}(t) & p_{13}(t) \\ p_{21}(t) & p_{22}(t) & p_{23}(t) \\ p_{31}(t) & p_{32}(t) & p_{33}(t)\end{array}\right)$ :

$$
\begin{array}{r}
p_{11}(t)=\int_{\triangle_{m}(t)} d x, \\
p_{12}(t)=p_{21}(t)=\int_{\triangle_{m}(t)} \xi_{m}(t) d x, \\
p_{13}(t)=p_{31}(t)=\int_{\triangle_{m}(t)} \eta_{m}(t) d x, \\
p_{22}(t)=\int_{\triangle_{m}(t)} \xi_{m}(t)^{2} d x, \\
p_{23}(t)=p_{32}(t)=\int_{\triangle_{m}(t)} \xi_{m}(t) \eta_{m}(t) d x, \\
p_{33}(t)=\int_{\triangle_{m}(t)} \eta_{m}(t)^{2} d x .
\end{array}
$$

The matrix $Q_{m}(t)=\left(\begin{array}{lll}q_{11}(t) & q_{12}(t) & q_{13}(t) \\ q_{21}(t) & q_{22}(t) & q_{23}(t) \\ q_{31}(t) & q_{32}(t) & q_{33}(t)\end{array}\right)$ :

$$
\begin{gathered}
q_{11}(t)=q_{21}(t)=q_{31}(t)=0, \\
q_{12}(t)=\int_{\triangle_{m}(t)} \xi_{m}^{\prime}(t) d x, \\
q_{13}(t)=\int_{\triangle_{m}(t)} \eta_{m}^{\prime}(t) d x, \\
q_{22}(t)=\int_{\triangle_{m}(t)} \xi_{m}(t) \xi_{m}^{\prime}(t) d x, \\
q_{23}(t)=\int_{\triangle_{m}(t)} \xi_{m}(t) \eta_{m}^{\prime}(t) d x, \\
q_{32}(t)=\int_{\triangle_{m}(t)} \eta_{m}(t) \xi_{m}^{\prime}(t) d x, \\
q_{33}(t)=\int_{\triangle_{m}(t)} \eta_{m}(t) \eta_{m}^{\prime}(t) d x .
\end{gathered}
$$

The matrix $K_{m}(t)=\left(\begin{array}{lll}k_{11}(t) & k_{12}(t) & k_{13}(t) \\ k_{21}(t) & k_{22}(t) & k_{23}(t) \\ k_{31}(t) & k_{32}(t) & k_{33}(t)\end{array}\right)$ : 


$$
\begin{array}{r}
k_{11}(t)=\int_{\triangle_{m}(t)} \nabla \cdot \vec{a} d x, \\
k_{12}(t)=k_{21}(t)=\int_{\triangle_{m}(t)} \xi_{m}(t) \nabla \cdot \vec{a} d x, \\
k_{13}(t)=k_{31}(t) \int_{\triangle_{m}(t)} \eta_{m}(t) \nabla \cdot \vec{a} d x, \\
k_{22}(t)=\int_{\triangle_{m}(t)} \xi_{m}(t)^{2} \nabla \cdot \vec{a} d x, \\
k_{23}(t)=k_{32}(t) \int_{\triangle_{m}(t)} \xi_{m}(t) \eta_{m}(t) \nabla \cdot \vec{a} d x, \\
k_{33}(t)=\int_{\triangle_{m}(t)} \eta_{m}(t)^{2} \nabla \cdot \vec{a} d x .
\end{array}
$$

The matrix $W_{m}(t)=\left(\begin{array}{lll}w_{a m 1}(t) & w_{b m 1}(t) & w_{c m 1}(t) \\ w_{a m 2}(t) & w_{b m 2}(t) & w_{c m 2}(t) \\ w_{a m 3}(t) & w_{b m 3}(t) & w_{c m 3}(t)\end{array}\right)$ :

$$
\begin{gathered}
w_{a m 1}(t)=\sum_{l=i, j, k}\left(-\beta r_{1 l}\right), \\
w_{b m 1}(t)=\sum_{l=i, j, k}\left(\frac{r_{1 l} n_{l, x}}{2 h_{m}}-\beta r_{2 l m}\right), \\
w_{c m 1}(t)=\sum_{l=i, j, k}\left(\frac{r_{1 l} n_{l, y}}{2 h_{m}}-\beta r_{3 l m}\right), \\
w_{a m 2}(t)=\sum_{l=i, j, k}\left(-\frac{r_{1 l} n_{l, x}}{2 h_{m}}-\beta r_{2 l m}\right), \\
w_{b m 2}(t)=\sum_{l=i, j, k}\left(-\beta s_{1 m m l}\right), \\
w_{c m 2}(t)=\sum_{l=i, j, k}\left(\frac{r_{2 l m} n_{l, y}-r_{3 l m} n_{l, x}}{2 h_{m}}-\beta s_{2 m m l}\right), \\
w_{a m 3}(t)=\sum_{l=i, j, k}\left(-\frac{r_{1 l} n_{l, y}}{2 h_{m}}-\beta r_{3 l m}\right), \\
w_{b m 3}(t)=\sum_{l=i, j, k}\left(\frac{r_{3 l m} n_{l, x}-r_{2 l m} n_{l, y}}{2 h_{m}}-\beta s_{2 m m l}\right), \\
w_{c m 3}(t)=\sum_{l=i, j, k}\left(-\beta s_{3 m m l}\right) .
\end{gathered}
$$




$$
\begin{array}{r}
\text { The matrix } W_{l}(t)=\left(\begin{array}{rrr}
w_{a l 1}(t) & w_{b l 1}(t) & w_{c l 1}(t) \\
w_{a l 2}(t) & w_{b l 2}(t) & w_{c l 2}(t) \\
w_{a l 3}(t) & w_{b l 3}(t) & w_{c l 3}(t)
\end{array}\right), l=i, j, k: \\
w_{a l 1}(t)=\beta r_{1 l}, \\
w_{b l 1}(t)=\frac{r_{1 l} n_{l, x}}{2 h_{l}}+\beta r_{2 l}, \\
w_{c l 1}(t)=\frac{r_{1 l} n_{l, y}}{2 h_{l}}+\beta r_{3 l}, \\
w_{a l 2}(t)=-\frac{r_{1 l} n_{l, x}}{2 h_{m}}+\beta r_{2 l m}, \\
w_{b l 2}(t)=\frac{r_{2 l m} n_{l, x}}{2 h_{l}}-\frac{r_{2 l} n_{l, x}}{2 h_{m}}+\beta s_{1 l m}, \\
w_{c l 2}(t)=\frac{r_{2 l m} n_{l, y}}{2 h_{l}}-\frac{r_{3 l} n_{l, x}}{2 h_{m}}+\beta s_{2 l m}, \\
w_{a l 3}(t)=-\frac{r_{1 l} n_{l, y}}{2 h_{m}}+\beta r_{3 l m}, \\
w_{b l 3}(t)=\frac{r_{3 l m} n_{l, x}}{2 h_{l}}-\frac{r_{2 l} n_{l, y}}{2 h_{m}}+\beta s_{3 l m}, \\
w_{c l 3}(t)=\frac{r_{3 l m} n_{l, y}}{2 h_{l}}-\frac{r_{3 l} n_{l, y}}{2 h_{m}}+\beta s_{4 l m},
\end{array}
$$

where

$$
\begin{array}{r}
r_{1 l}=\int_{e_{l}(t)} d S, r_{2 l}=\int_{e_{l}(t)} \xi_{l}(t) d S, r_{3 l}=\int_{e_{l}(t)} \eta_{l}(t) d S, \\
r_{2 l m}=\int_{e_{l}(t)} \xi_{m}(t) d S, r_{3 l m}=\int_{e_{l}(t)} \eta_{m}(t) d S, \\
s_{1 m m l}=\int_{e_{l}(t)} \xi_{m}(t)^{2} d S, s_{2 m m l}=\int_{e_{l}(t)} \xi_{m}(t) \eta_{m}(t) d S, \\
s_{3 m m l}=\int_{e_{l}(t)} \eta_{m}(t)^{2} d S, s_{1 l m}=\int_{e_{l}(t)} \xi_{m}(t) \xi_{l}(t) d S, \\
s_{2 l m}=\int_{e_{l}(t)} \xi_{m}(t) \eta_{l}(t) d S, s_{3 l m}=\int_{e_{l}(t)} \xi_{l}(t) \eta_{m}(t) d S, s_{4 l m}=\int_{e_{l}(t)} \eta_{m}(t) \eta_{l}(t) d S .
\end{array}
$$

\section{References}

[1] M. Alber, H.G.E. Hentschel, B. Kazmierczak, S.A. Newman. Existence of solutions to a new model of biological pattern formation. J. Math. Anal. Appl., 308 (2005), No. 1, 175-194.

[2] M. Alber, T. Glimm, H.G.E. Hentschel, B. Kazmierczak, Y.-T. Zhang, J. Zhu, S.A. Newman. The morphostatic limit for a model of skeletal pattern formation in the vertebrate limb. Bulletin of Mathematical Biology, 70 (2008), No. 2, 460-483. 
[3] Y. Cheng, C.-W. Shu. A discontinuous Galerkin finite element method for time dependent partial differential equations with higher order derivatives. Mathematics of Computation, 77 (2008), No. 262, 699-730.

[4] B. Cockburn, G. Karniadakis, C.-W. Shu. The development of discontinuous Galerkin methods, in Discontinuous Galerkin Methods: Theory, Computation and Applications, B. Cockburn, G. Karniadakis, and C.-W. Shu, Editors. Lecture Notes in Computational Science and Engineering, 11 (2000), Springer, 3-50.

[5] B. Cockburn, C.-W. Shu. Runge-Kutta discontinuous Galerkin methods for convectiondominated problems. Journal of Scientific Computing, 16 (2001), No. 3, 173-261.

[6] B. Cockburn, C.-W. Shu. The local discontinuous Galerkin method for time-dependent convection-diffusion systems. SIAM Journal on Numererical Analysis, 35 (1998), No. 6, 2440-2463.

[7] H.G.E. Hentschel, T. Glimm, J.A. Glazier, S.A. Newman. Dynamical mechanisms for skeletal pattern formation in the vertebrate limb. Proc. R. Soc. B, 271 (2004), No. 1549, 1713-1722.

[8] W. Hundsdorfer. Trapezoidal and midpoint splittings for initial-boundary value problems. Mathematics of Computation, 67 (1998), No. 223, 1047-1062.

[9] P.K. Kundu. Fluid Mechanics. Academic Press, Inc, London, 1990.

[10] D. Levy, C.-W. Shu, J. Yan. Local discontinuous Galerkin methods for nonlinear dispersive equations. Journal of Computational Physics, 196 (2004), No. 2, 751-772.

[11] A. Madzvamuse, A.J. Wathen, P.K. Maini. A moving grid finite element method applied to a model biological pattern generator. Journal of Computational Physics, 190 (2003), No. 2, 478-500.

[12] A. Madzvamuse, P.K. Maini, A.J. Wathen. A moving grid finite element method for the simulation of pattern generation by Turing models on growing domains. J. Sci. Comput., 24 (2005), No. 2, 247-262.

[13] A. Madzvamuse. Time-stepping schemes for moving grid finite elements applied to reactiondiffusion systems on fixed and growing domains. Journal of Computational Physics, 214 (2006), No. 1, 239-263.

[14] C.E. Nelson, B.A. Morgan, A.C. Burke, E. Laufer, E. DiMambro, L.C. Murtaugh, E. Gonzales, L. Tessarollo, L.F. Parada, C. Tabin. Analysis of Hox gene expression in the chick limb bud. Development, 122 (1996), No. 5, 1449-1466.

[15] S.A. Newman, G.B. Müller. Origination and innovation in the vertebrate limb skeleton: an epigenetic perspective. J. Exp. Zoolog. B Mol. Dev. Evol. 304 (2005), No. 6, 593-609. 
[16] S.A. Newman, R. Bhat. Activator-inhibitor dynamics of vertebrate limb pattern formation. Birth Defects Res C Embryo Today, 81 (2007), No. 4, 305-319.

[17] S.A. Newman, S. Christley, T. Glimm, H.G.E. Hentschel, B. Kazmierczak, Y.-T. Zhang, J. Zhu, M. Alber. Multiscale models for vertebrate limb development. Curr. Top. Dev. Biol., 81 (2008), 311-340.

[18] M.A. Ros, G.E. Lyons, S. Mackem, J.F. Fallon. Recombinant limbs as a model to study homeobox gene regulation during limb development. Dev. Biol., 166 (1994), No. 1, 59-72.

[19] G. Strang. On the construction and comparison of difference schemes. SIAM J. Numer. Anal., 8 (1968), No. 3, 506-517.

[20] D. Summerbell. A descriptive study of the rate of elongation and differentiation of the skeleton of the developing chick wing. J. Embryol. Exp. Morphol., 35 (1976), No. 2, 241-260.

[21] T. Svingen, K.F. Tonissen. Hox transcription factors and their elusive mammalian gene targets. Heredity, 97 (2006), No. 2, 88-96.

[22] C. Tickle. Patterning systems - from one end of the limb to the other. Dev. Cell, 4 (2003), No. 4, 449-458.

[23] Y. Xu, C.-W. Shu. Local discontinuous Galerkin methods for three classes of nonlinear wave equations. Journal of Computational Mathematics, 22 (2004), No. 2, 250-274.

[24] Y. Xu, C.-W. Shu. Local discontinuous Galerkin methods for nonlinear Schrodinger equations. Journal of Computational Physics, 205 (2005), No. 1, 72-97.

[25] Y. Xu, C.-W. Shu. Local discontinuous Galerkin methods for two classes of two dimensional nonlinear wave equations. Physica D, 208 (2005), No. 1-2, 21-58.

[26] Y. Xu, C.-W. Shu. Local discontinuous Galerkin methods for the Kuramoto-Sivashinsky equations and the Ito-type coupled KdV equations. Computer Methods in Applied Mechanics and Engineering, 195 (2006), No. 25-28, 3430-3447.

[27] J. Yan, C.-W. Shu. A local discontinuous Galerkin method for KdV type equations. SIAM Journal on Numerical Analysis, 40 (2002), No. 2, 769-791.

[28] J. Yan, C.-W. Shu. Local discontinuous Galerkin methods for partial differential equations with higher order derivatives. Journal of Scientific Computing, 17 (2002), No. 1-4, 27-47.

[29] J. Zhu, Y.-T. Zhang, S.A. Newman, M. Alber. Application of discontinuous Galerkin methods for reaction-diffusion systems in developmental biology. Journal of Scientific Computing, 40 (2009), No. 1-3, 391-418.

[30] E. Zwilling. Development of fragmented and of dissociated limb bud mesoderm. Dev. Biol., 9 (1964), No. 1, 20-37. 